\title{
Hemogasometria em cães com desidratação experimental tratados com soluções eletrolíticas comerciais administradas por via intravenosa
}

\author{
Blood gas analysis in dogs with experimental dehidration treated with commercial electrolytes \\ solutions by intravenous route
}

\author{
José Dantas Ribeiro Filho ${ }^{I^{*}}$ Paula de Zorzi Balbinot ${ }^{\mathrm{I}}$ José Antônio Viana $^{\mathrm{I}}$ \\ Waleska de Melo Ferreira Dantas ${ }^{\mathrm{I}}$ Betânia Souza Monteiro ${ }^{\mathrm{I}}$
}

\section{RESUMO}

No presente estudo, foram comparados os efeitos da administração intravenosa de três soluções eletrolíticas comerciais sobre o equilíbrio ácido-base em cães desidratados experimentalmente por restrição hídrica e poliúria. Os animais foram aleatoriamente distribuídos em três grupos e tratados com três diferentes soluções eletrolíticas comerciais durante 12 horas: Ringer com lactato de sódio (RL), Ringer simples (RS) e Glicofisiológico (GF). Entre os tratamentos testados, a fluidoterapia intravenosa com solução de Ringer com lactato de sódio $(R L)$ foi o tratamento que apresentou efeito alcalinizante, sinalizado por pequeno aumento nos valores do pH(a), $\mathrm{CHCO}_{3}(\mathrm{aP}), \mathrm{ctCO}_{2}(\mathrm{aP})$ e cBase(a), podendo ser utilizada no tratamento de animais com acidose metabólica de intensidade discreta a moderada. As soluções Ringer simples (RS) e glicofisiológica (GF) determinaram discreta diminuição na concentração de base titulável do sangue arterial (cBase), demonstrando efeito acidificante, o que as tornam uma opção para tratar cães com alcalose metabólica.

Palavras-chave: cães, hemogasometria, hidratação, cristalóides, desidratação.

\section{ABSTRACT}

Three commercial intravenous electrolyte solutions were compared as for their effects on the blood acid-base status in dogs experimentally dehydrated by withholding water and inducing polyuria. Animals were randomly divided into three groups which were rehydrated with the following commercial electrolyte solutions during 12 hours: Lactate Ringer's solution $(R L)$, Ringer's solution (RS) and a normal saline solution $(0.9 \%$ sodium chloride) containing 5\% dextrose (GF). The RL's intravenous fluid therapy resulted in an alkalinizing effect demonstrated by a mild increase in arterial blood $\mathrm{pH}, \mathrm{ctCO}_{2}$, bicarbonate ( $\mathrm{CHCO}_{3}^{-}$), and arterial blood base concentration (cBase) and, thus, can be used in animals exhibiting mild to moderate metabolic acidosis. In contrast, the RS and GF therapies led to a mild decrease in the concentration of arterial blood tritiable base (cBase) inducing an acidifying effect, which make them an option to treat dogs with metabolic alkalosis.

Key words: dogs, blood gas analysis, fluid therapy, crystalloids, dehydration.

\section{INTRODUÇÃO}

A desidratação é uma das desordens mais comuns na prática da clínica médico-veterinária. Essa alteração usualmente está presente na maioria das enfermidades que acometem os cães, sendo, muitas vezes, problemas secundários a uma doença base. A correção da desidratação é feita por meio da fluidoterapia, uma das medidas terapêuticas mais importantes e mais freqüentemente utilizadas em medicina intensiva, a qual é indicada na recuperação e na manutenção da perfusão e de funções celulares, corrigindo desequilíbrios hidroeletrolíticos e ácido-base e, conseqüentemente, a homeostase.

O tratamento dos distúrbios hidroeletrolíticos e ácido-base é freqüentemente empírico, baseado na literatura, na opinião e na recomendação de especialistas. Os fluidos rotineiramente utilizados no tratamento desses distúrbios são os cristalóides e os colóides. Os cristalóides são soluções que contêm solutos eletrolíticos e não-eletrolíticos capazes de penetrar em todos os compartimentos corporais (p. ex. Ringer com lactato de sódio, solução de $\mathrm{NaCl}$ a $0,9 \%$, glicose). São

'Departamento de Veterinária, Universidade Federal de Viçosa (DVT/UFV). Av. P. H. Rolfs, s/n, Centro, 36570-000, Viçosa, MG,

Brasil. E-mail: dantas@ufv.br. *Autor para correspondência. 
classificados em soluções de reposição e de manutenção. As de reposição apresentam composição de eletrólitos similar à do plasma, tendo características semelhantes às do fluido extracelular. Já as de manutenção são compostas por menos sódio e mais potássio do que as de reposição (FERREIRA \& PACHALY 2000; CONSTABLE 2003; MATHEWS 2006).

Os fluidos de reposição são isotônicos, alcalinizantes ou acidificantes, tendo como base o sódio na sua constituição. As soluções acidificantes são $\mathrm{NaCl}$ $0,9 \%$, glicose $5 \%$ e Ringer simples. As alcalinizantes contêm precursores de bicarbonato para prevenir acidemia como o lactato (Ringer com lactato), acetato e gluconato (MATHEWS, 1998; FERREIRA \& PACHALY, 2000; MATHEWS, 2006). O tipo de solução escolhida influencia na correção ou na indução de uma acidose ou uma alcalose, portanto, se feita de forma inapropriada, pode agravar o quadro clínico do animal, por isso, a escolha criteriosa da solução adequada para cada tipo de desordem é uma conduta importante.

O conhecimento do clínico sobre homeostase, desequilíbrios hidroeletrolíticos e ácidobase reflete-se na escolha de soluções eletrolíticas adequadas para a correção desses desequilíbrios. Isso se traduz na recuperação mais eficiente dos pacientes que diariamente chegam à clínica, o que faz da fluidoterapia um recurso terapêutico imprescindível para esses animais (FOSTER, 1970). Por isso, faz-se necessário avaliar e quantificar a eficácia terapêutica de soluções hidratantes disponíveis comercialmente para uso parenteral, buscando-se caracterizar os seus efeitos na recomposição da homeostase (LINDEMAN \& PAPPER, 1975). O presente estudo teve como objetivo avaliar os efeitos da administração intravenosa de três soluções eletrolíticas comerciais sobre o equilíbrio ácido-base em cães desidratados experimentalmente por restrição hídrica e poliúria.

\section{MATERIAL E MÉTODOS}

Foram utilizados seis cães, machos adultos, sem raça definida, pesando entre 5-15kg, clinicamente hígidos. Antes do início do experimento os cães foram submetidos a exame físico, à hemograma, à urinálise, à perfil bioquímico e à pesquisa de hematozoários. Os animais foram cedidos pela Sociedade Protetora dos Animais de Viçosa (SOVIPA), ficando aos cuidados de um veterinário responsável e foram alojados em baias coletivas. Foi fornecida ração comercial ${ }^{a}$ duas vezes ao dia e água ad libitum.

O modelo de desidratação foi desenvolvido para padronização da desidratação com finalidade de avaliar o potencial terapêutico das soluções reidratantes por meio de jejum hídrico e alimentar de 24 horas e aplicação de furosemida ${ }^{\mathrm{b}}$ administrada por via intravenosa em três dosagens de $4 \mathrm{mg} \mathrm{kg}^{-1}$ com intervalo de 8 horas. A fase de indução da desidratação teve duração de 24 horas e logo após este período, foram realizados os tratamentos a seguir: o grupo RL recebeu solução eletrolítica isotônica intravenosa de Ringer com lactato de sódioc; o grupo RS recebeu solução eletrolítica isotônica intravenosa de Ringer simplesc, enquanto o grupo GF recebeu solução eletrolítica intravenosa de soro glicofisiológico ${ }^{c}$. O volume de soro administrado aos animais de todos os grupos foi baseado na fórmula: peso corporal x grau de desidratação + taxa de manutenção $\left(25 \mathrm{~mL} \mathrm{~kg}^{-1} 12 \mathrm{~h}^{-1}\right)$.

Para a análise hemogasométrica, amostras de sangue arterial foram coletadas anaerobicamente da artéria femoral em seringas plásticas descartáveis ${ }^{\mathrm{d}}$ de $3 \mathrm{~mL}$, previamente heparinizadas ${ }^{\mathrm{e}}$ e acondicionadas em água com gelo e encaminhadas imediatamente para análise das seguintes variáveis: $\mathrm{pH}$ do sangue arterial - pH(a), pressão parcial de oxigênio do sangue arterial - $\mathrm{pO}_{2}(\mathrm{a})$, pressão parcial do dióxido de carbono no plasma do sangue arterial - $\mathrm{pCO}_{2}(\mathrm{a})$, concentração total do dióxido de carbono no plasma do sangue arterial $\mathrm{ctCO}_{2}(\mathrm{aP})$, concentração de bicarbonato no plasma do sangue arterial - $\mathrm{CHCO}_{3}^{-}(\mathrm{aP})$, concentração de base titulável do sangue arterial - cBase(a) e saturação de oxigênio do sangue arterial - $\mathrm{sO}_{2}(\mathrm{a})$. Essas análises foram realizadas em aparelho de hemogasometria ${ }^{\mathrm{f}} \mathrm{com}$ calibração automática, segundo os intervalos: T0 (imediatamente antes do início da desidratação - 0h), T1 (final do período de desidratação - 24h), T2 (seis horas de hidratação - 6h), T3 (12 horas de hidratação 12h - término do período de hidratação) e T4 (12 horas após o término de T3 - correspondente à fase de avaliação final).

O sangue coletado por meio de venopunção jugular direta, em tubos sem anticoagulanteg, foi utilizado para mensuração de sódio, potássio e cloreto. A mensuração de sódio e potássio foi realizada por espectofotometria de chamas, em aparelho espectofotômetro ${ }^{\mathrm{h}}$. A mensuração do cloreto foi realizada por meio de um analisador automático de bioquímicai. Com os resultados da bioquímica sérica e hemogasometria, foi calculado, o Ânion Gap $=\left(\mathrm{Na}^{+}+\right.$ $\left.\mathrm{K}^{+}\right)-\left(\mathrm{Cl}^{-}+\mathrm{HCO}_{3}^{-}\right)$segundo MORAIS \& CONSTABLE (2006) e WELLMAN et al. (2006).

O delineamento experimental foi inteiramente casualizado. Os animais foram distribuídos aleatoriamente em dois quadrados latinos $3 \mathrm{X} 3$, em esquema de parcelas subdivididas, sendo que os tratamentos representam as parcelas e os tempos de avaliação representam as 
subparcelas, com período de nove dias, sendo sete dias de adaptação e dois dias de tratamento.

Para avaliar o efeito dos tratamentos, foi utilizada a análise de variância baseada em planejamento de medidas repetidas, ou seja, foi avaliado cada tratamento em vários tempos de acompanhamento. Além de avaliada a influência do tratamento, foi analisada a influência do tempo e a interação entre o tratamento e o tempo. Quando a análise foi significativa para um ou mais fatores, foi utilizado o teste de comparações múltiplas de médias LSD (Least Significant Difference) para avaliar o efeito. Todos os resultados foram considerados significativos quando $\mathrm{P}<0,05$.

\section{RESULTADOS E DISCUSSÃO}

Os resultados dos valores do $\mathrm{pH}(\mathrm{a}), \mathrm{pO}_{2}(\mathrm{a})$, $\mathrm{pCO}_{2}(\mathrm{a}), \mathrm{cHCO}_{3}(\mathrm{aP}), \mathrm{ctCO}_{2}(\mathrm{aP}), \mathrm{cBase}(\mathrm{a}), \mathrm{sO}_{2}(\mathrm{a})$ e ânion gap (AG) estão expressos na tabela 1 .

Tabela 1 - pH(a), $\mathrm{pO}_{2}(\mathrm{a}), \mathrm{pCO}_{2}(\mathrm{a}), \mathrm{cHCO}_{3}(\mathrm{aP}), \mathrm{ctCO}_{2}(\mathrm{aP})$, cBase(a), sO $\mathrm{S}_{2}(\mathrm{a})$ e ânion gap sangüíneos em cães com desidratação induzida e tratados com solução de Ringer lactato (RL), Ringer simples (RS) e Glicoficofisiológico (GF) por via IV em diferentes tempos.

\begin{tabular}{|c|c|c|c|c|c|}
\hline Tratamento & T0 (0hi) & T1 (24hi) & T2 (6ht) & T3 (12ht) & T4 (12h av. f.) \\
\hline \multicolumn{6}{|c|}{ pH(a) média \pm desvio padrão } \\
\hline RL & $7,40 \pm 0,04^{\text {a в }}$ & $7,42 \pm 0,05^{\text {a } \mathrm{B}}$ & $7,45 \pm 0,03^{\text {a A }}$ & $7,44 \pm 0,03^{\text {a A }}$ & $7,42 \pm 0,03^{\text {a A,B }}$ \\
\hline RS & $7,39 \pm 0,03^{\mathrm{aA}}$ & $7,42 \pm 0,06^{\mathrm{a} A}$ & $7,40 \pm 0,03^{\mathrm{b} \mathrm{A}}$ & $7,40 \pm 0,04^{\mathrm{bA}}$ & $7,42 \pm 0,03^{\mathrm{a} \mathrm{A}}$ \\
\hline GF & $7,39 \pm 0,04^{\text {a A }}$ & $7,42 \pm 0,04^{\text {a A }}$ & $7,41 \pm 0,03^{\mathrm{bA}}$ & $7,42 \pm 0,03^{\mathrm{bA}}$ & $7,41 \pm 0,03^{\mathrm{aA}}$ \\
\hline \multicolumn{6}{|c|}{$\mathrm{pO}_{2}$ (a) média \pm desvio padrão } \\
\hline RL & $87,3 \pm 10,0^{\text {a A }}$ & $76,5 \pm 10,34^{\text {a A }}$ & $88,3 \pm 18,46^{\text {a A }}$ & $88,1 \pm 23,29^{\text {a A }}$ & $83,1 \pm 9,66^{\text {a A }}$ \\
\hline RS & $87,5 \pm 3,14^{\mathrm{aA}}$ & $90,1 \pm 24,04^{\text {a A }}$ & $87,5 \pm 12,51^{\text {a A }}$ & $81,0 \pm 16,55^{\text {a A }}$ & $82,8 \pm 16,26^{\text {a } A}$ \\
\hline GF & $88,5 \pm 7,94^{\mathrm{a} \mathrm{A}}$ & $89,3 \pm 27,60^{\text {a A }}$ & $94,1 \pm 21,75^{\mathrm{aA}}$ & $88,1 \pm 13,61^{\text {a A }}$ & $85,6 \pm 17,63^{\text {a A }}$ \\
\hline \multicolumn{6}{|c|}{$\mathrm{pCO}_{2}(\mathrm{a})$ média \pm desvio padrão } \\
\hline RL & $31,0 \pm 1,67^{\text {а в }}$ & $34,5 \pm 2,94^{\text {a A }}$ & $34,3 \pm 2,33^{\text {a A,B }}$ & $34,2 \pm 2,56^{\text {a A,B }}$ & $35,2 \pm 2,92^{\text {a A,B }}$ \\
\hline RS & $32,3 \pm 1,75^{\text {а в }}$ & $32,5 \pm 5,05^{\text {a A }}$ & $32,2 \pm 3,87^{\text {a A,B }}$ & $32,3 \pm 3,07^{\text {a A,B }}$ & $31,8 \pm 4,49^{\text {a A,B }}$ \\
\hline GF & $32,5 \pm 5,0^{\mathrm{a} \mathrm{B}}$ & $36,1 \pm 3,92^{\text {a A }}$ & $34,3 \pm 3,61^{\text {a A,B }}$ & $32,5 \pm 3,61^{\text {а А,B }}$ & $32,8 \pm 3,54^{\text {a A,B }}$ \\
\hline \multicolumn{6}{|c|}{$\mathrm{cHCO}_{3}^{-}(\mathrm{aP})$ média \pm desvio padrão } \\
\hline RL & $18,83 \pm 2,14^{\text {a C }}$ & $21,67 \pm 2,94^{\mathrm{aB}, \mathrm{C}}$ & $23,50 \pm 1,38^{\text {a A }}$ & $23,0 \pm 1,55^{\text {a A }}$ & $22,83 \pm 1,60^{\text {a B }}$ \\
\hline RS & $19,50 \pm 1,87^{\mathrm{a} \mathrm{A}}$ & $20,50 \pm 4,23^{\text {a A }}$ & $19,33 \pm 1,21^{\mathrm{bA}}$ & $19,83 \pm 0,98^{\mathrm{b} \mathrm{A}}$ & $21,17 \pm 1,83^{\text {a A }}$ \\
\hline GF & $19,0 \pm 2,75^{\text {а в }}$ & $23,0 \pm 2,19^{\text {a A }}$ & $21,5 \pm 2,81^{\mathrm{a}, \mathrm{b} A, \mathrm{~B}}$ & $20,33 \pm 2,34^{\text {b A,B }}$ & $20,5 \pm 1,87^{\text {a A,B }}$ \\
\hline \multicolumn{6}{|c|}{$\mathrm{ctCO}_{2}(\mathrm{aP})$ média \pm desvio padrão } \\
\hline RL & $19,83 \pm 2,14^{\text {a B }}$ & $22,83 \pm 2,71^{\mathrm{a}, \mathrm{b} A, \mathrm{~B}}$ & $24,5 \pm 1,38^{\text {a A }}$ & $24,33 \pm 1,21^{\text {a A }}$ & $23,83 \pm 1,60^{\text {a } A}$ \\
\hline RS & $20,5 \pm 1,87^{\text {a A }}$ & $21,66 \pm 4,46^{\text {b A }}$ & $20,5 \pm 1,38^{\mathrm{bA}}$ & $20,83 \pm 0,98^{\mathrm{bA}}$ & $21,83 \pm 2,31^{\text {a A }}$ \\
\hline GF & $20,16 \pm 2,86^{\text {a B }}$ & $24,16 \pm 2,48^{\text {a A }}$ & $22,5 \pm 2,81^{\mathrm{a}, \mathrm{b} \mathrm{A}} \mathrm{B}$ & $21,34 \pm 2,34^{\text {b A,B }}$ & $21,5 \pm 1,87^{\text {а А.,B }}$ \\
\hline \multicolumn{6}{|c|}{ cBase(a) média \pm desvio padrão } \\
\hline RL & $-4.33 \pm 2,42^{\text {a } \mathrm{B}}$ & $-1,66 \pm 3,20$ а в & $0,66 \pm 1,63^{\text {a A }}$ & $0,50 \pm 1,64^{\mathrm{aA}}$ & $-0,50 \pm 1,51^{\text {a A }}$ \\
\hline RS & $-4,33 \pm 2,34^{\text {a A }}$ & $-2,50 \pm 4,32^{\text {a A }}$ & $-3,83 \pm 1,17^{\mathrm{bA}}$ & $-3,16 \pm 1,33^{\mathrm{bA}}$ & $-2,0 \pm 1,26^{\text {a A }}$ \\
\hline GF & $-4,50 \pm 2,43^{\text {a } \mathrm{B}}$ & $-0,33 \pm 2,25^{\text {a A }}$ & $-1,66 \pm 2,5^{\mathrm{a}, \mathrm{b} A, \mathrm{~B}}$ & $-2,66 \pm 1,97^{\mathrm{a}, \mathrm{b}} \mathrm{A}, \mathrm{B}$ & $-2,83 \pm 1,6^{\text {a A,B }}$ \\
\hline \multicolumn{6}{|c|}{$\mathrm{sO}_{2}(\mathrm{a})$ média \pm desvio padrão } \\
\hline RL & $96,5 \pm 1,04^{\mathrm{a} \mathrm{A}}$ & $94,8 \pm 1,83^{\text {a A }}$ & $96,6 \pm 1,63^{\text {a A }}$ & $96,0 \pm 2,75^{\mathrm{a} \mathrm{A}}$ & $96,0 \pm 1,67^{\text {a A }}$ \\
\hline RS & $96,3 \pm 0,51^{\text {a A }}$ & $96,3 \pm 1,75^{\text {a A }}$ & $96,3 \pm 2,25^{\text {a A }}$ & $95,1 \pm 2,04^{\text {a A }}$ & $95,8 \pm 2,31^{\text {a A }}$ \\
\hline GF & $96,3 \pm 1,21^{\text {a A }}$ & $95,5 \pm 3,14^{\text {a A }}$ & $96,6 \pm 1,36^{\text {a A }}$ & $96,1 \pm 2,13^{\text {a A }}$ & $95,6 \pm 2,87^{\text {a A }}$ \\
\hline \multicolumn{6}{|c|}{ Ânion Gap média \pm desvio padrão } \\
\hline $\mathrm{RL}$ & $7,88 \pm 14,89^{\text {a A,B }}$ & $14,21 \pm 11,38^{\text {a A }}$ & $3,55 \pm 11,81^{\text {a } \mathrm{B}}$ & $2,66 \pm 14,72^{\text {а в }}$ & $4,35 \pm 9,40^{\text {a B }}$ \\
\hline RS & $11,58 \pm 6,46^{\text {a A,B }}$ & $20,45 \pm 5,93^{\text {a A }}$ & $11,38 \pm 6,06^{\text {a B }}$ & $7,23 \pm 19,04^{\text {a B }}$ & $5,13 \pm 12,21^{\text {a } \mathrm{B}}$ \\
\hline GF & $10,96 \pm 11,15^{\text {a A,B }}$ & $12,76 \pm 10,76^{\text {a A }}$ & $6,38 \pm 8,75^{\text {а в }}$ & $8,16 \pm 8,42^{\text {а в }}$ & $9,03 \pm 15,16^{\text {а в }}$ \\
\hline
\end{tabular}

Análise de variância (medidas repetidas).

As médias na mesma coluna seguidas por letras minúsculas diferentes e as médias na mesma linha seguidas por letras maiúsculas diferentes diferem pelo teste de Turkey $(\mathrm{P}<0,05)$.

T0 - T1 = período de indução da desidratação (24 horas); T2 - T3 = período de tratamento (12 horas); T4 = período de avaliação final (12 horas após término do tratamento).

$\mathrm{RL}=$ Ringer com lactato de sódio, $\mathrm{RS}=$ Ringer simples e GF = Glicofisiológico foram administrados via IV . O volume infundido foi baseado na fórmula: peso corporal x grau de desidratação + taxa de manutenção (25mL kg $\left.\mathrm{kg}^{-1} 12 \mathrm{~h}^{-1}\right)$.

Ciência Rural, v.38, n.7, out, 2008. 
Na fase final de indução da desidratação (T1), constatou-se discreto aumento dos valores do pH sangüíneo nos animais dos três tratamentos, porém, sem diferença significativa $(\mathrm{P}=0,323)$. Este aumento foi ocasionado pela administração da furosemida, substância utilizada na potencialização da desidratação nos animais. Para corrigir a hipovolemia, o organismo aumenta a reabsorção de sódio. Entretanto, para manter a eletroneutralidade, há necessidade que a reabsorção de sódio no túbulo proximal seja acompanhada pela reabsorção de ânions como o cloreto. Como o paciente está com hipocloremia, maior quantidade de $\mathrm{Na}^{+}$alcança o túbulo distal, onde, sob a ação da aldosterona, estimula a secreção de $\mathrm{H}^{+}$para reter $\mathrm{Na}^{+}$. Porém, a excreção renal de $\mathrm{H}^{+}$é diretamente proporcional à reabsorção de bicarbonato, sobrevindo a alcalinemia (FREESTONE etal., 1989; BREYER \& JACOBSON 1990; RIBEIRO FILHO et al., 2007).

Em T2 e T3, fase em que os animais estavam em fluidoterapia, houve diferença entre o tratamento $\mathrm{RL}(\mathrm{P}=0,0001)$, que apresentou valores maiores de $\mathrm{pH}$, demonstrando uma alcalinemia (Tabela 1). Ringer com lactato de sódio é uma solução cristalóide isotônica, balanceada, poliônica e alcalinizante que é utilizado como expansor plasmático e na correção de acidose metabólica (MATHEUS, 1998 e MATHEWS, 2006). Essa ação tampão é decorrente do consumo de íons $\mathrm{H}^{+}$ durante o seu processo de transformação em glicose nos hepatócitos (NAYLOR \& FORSYTH, 1986) e de sua metabolização a bicarbonato (CONSTABLE, 2003). Os animais dos tratamentos RS e GF mantiveram os valores de $\mathrm{pH}$ sem diferença durante toda a fase experimental $(\mathrm{P}=0,520)$. Pelo fato de as soluções glicofisiológica e Ringer simples serem acidificantes (MATHEUS, 1998; CONSTABLE, 2003; DiBARTOLA, 2006), esperava-se que a administração dessas duas soluções ocasionasse efeito sobre os valores do $\mathrm{pH}$ sangüíneo, o que não ocorreu. Esse achado pode ter sido ocasionado pelo tempo de fluidoterapia empregado neste experimento, que foi de apenas 12 horas.

Os valores da $\mathrm{pO}_{2}$ não apresentaram diferença significativa entre os tratamentos, tampouco nos tratamentos ao longo do tempo $(\mathrm{P}=0,801)$, durante toda a fase experimental (Tabela 1). Os animais permaneceram com valores de $\mathrm{pO}_{2}$ na faixa de referência (80-103mmHg) descritos por KANEKO et al. (1997) e DiBARTOLA (2006), com exceção dos animais do RL no $\mathrm{T} 1$, que apresentaram valores inferiores aos de referência. Esse achado foi conseqüência da hipovolemia (RIBEIRO FILHO et al., 2007), que provavelmente, nos animais desse tratamento (RL) foi superior aos demais (RS e GF) como conseqüência de variação individual. O mesmo ocorreu com os valores da $\mathrm{pCO}_{2}$, ou seja, não houve interação entre tratamento e tempo, nem diferença significativa para esta variável $(\mathrm{P}=0,181)$. Entretanto, observou-se em T1 (Tabela 1) aumento discreto da $\mathrm{pCO}_{2}$ nos tratamentos RL e GF, porém os valores permaneceram dentro da faixa de normalidade, não podendo ser considerada uma acidose respiratória (KANEKO et al., 1997; DiBARTOLA, 2006). Possivelmente a causa para esse pequeno aumento tenha sido ocasionada pelo mecanismo de compensação, pois foi nesse tempo (T1) e nesses tratamentos (RL e GF) que também foram observados aumentos, embora discretos, nos valores de $\mathrm{ctCO}_{2}$, bicarbonato e cBase (Tabela 1), como citou JONSON (1995).

A concentração sérica de $\mathrm{HCO}_{3}^{-}$e a ctCO tiveram comportamentos semelhantes. No T1 houve aumento nas suas concentrações em todos os tratamentos, sem diferença significativa. Este aumento foi ocasionado pela administração da furosemida, substância utilizada na potencialização da desidratação nos animais (RIBEIRO FILHO et al., 2007) associado à alteração na $\mathrm{pCO}_{2}$ como descreveu JONHSON (1995).

Com o decorrer da fluidoterapia, ocorreram diferenças significativas entre os tratamentos em T2 e T3. No tratamento RL, houve aumento nas concentrações de $\mathrm{HCO}_{3}^{-}(\mathrm{P}=0,032)$ e na ctCO $(\mathrm{P}=0,043)$, demonstrando o efeito alcalinizante desta substância, confirmando as descrições de SEAHORN \& CORNICKSEAHORN (1994), MATHEUS (1998), CONSTABLE (2003) e MATHEWS (2006). Já nos tratamentos RS e GF, os valores destas variáveis diminuíram discretamente, voltando a estar semelhantes a T0 (Tabela 1), sinalizando a tendência acidificante dessas soluções e corroborando os relatos dos autores acima citados.

A concentração de base (cBase) reflete o componente metabólico de um distúrbio ácido-base, um valor negativo para cBase indica uma acidose metabólica enquanto um valor positivo indica alcalose metabólica (RUSSELL et al., 1996; DiBARTOLA, 2006). Após 24 horas da indução da desidratação (T1), observou-se pequeno aumento da cBase, porém, sem diferença significativa, exceto nos animais do grupo $\mathrm{GF}(\mathrm{P}=0,005)$. Esse aumento foi ocasionado devido à utilização de furosemida no protocolo de desidratação, mecanismo já descrito anteriormente. Possivelmente a diferença significativa observada nos animais do grupo GF foi determinada pela variação individual dos mesmos, pois todos os animais de todos os grupos receberam o mesmo protocolo de desidratação.

Em T2 e T3, durante a fase de hidratação, nos animais do grupo RL, houve um aumento significativo 
da cBase $(\mathrm{P}=0,028)$, demonstrando a ação alcalinizante da solução Ringer lactato (Tabela 1). LEAL et al. (2007), ao comparar a eficiência corretiva da acidose metabólica sistêmica por soluções contendo lactato-L ou bicarbonato em bovinos submetidos a quadro de acidose láctica ruminal aguda, concluiram que o lactato-L foi tão eficiente quanto o bicarbonato, podendo o mesmo ser utilizado como uma alternativa ao tratamento clássico com bicarbonato. Convém ressaltar que os referidos autores utilizaram uma solução contendo apenas o lactato-L. No presente estudo não foi constato efeito alcalinizante tão acentuado. Esse fato se deveu provavelmente ao uso da solução comercial Ringer lactato, que contém na sua composição 50\% de lactato$\mathrm{L}$ e $50 \%$ de lactato-D, traduzindo-se num efeito alcalinizante inferior, como citou CONSTABLE (2003). Grande parte do efeito tampão do lactato vem do consumo de um íons $\mathrm{H}^{+}$quando da oxidação do lactatoL ou de sua transformação em glicose nos hepatócitos (NAYLOR \& FORSYTH, 1986), embora muitos conceituem que a ação alcalinizante do lactato-L seja oriunda de sua transformação em bicarbonato (HARTSFIELD et al., 1981; KANEKO et al., 1997; RADOSTITS et al., 2002 e DiBARTOLA, 2006), esta afirmação é parcialmente verdadeira (NELSON \& COX, 2002). No caso da oxidação, os produtos finais são $\mathrm{CO}_{2}$ e $\mathrm{H}_{2} \mathrm{O}$, os quais podem ou não, dependendo de uma série de condições bioquímicas, ser transformados em bicarbonato e íons $\mathrm{H}^{+}$, pela ação da anidrase carbônica (NELSON \& COX, 2002).

Nos tratamentos RS e GF, ocorreu uma pequena diminuição da cBase (Tabela 1), evideciando a tendência acidificante das soluções glicofisiológica e Ringer simples, confirmando o que foi descrito anteriormente.

O valor de referência para $\mathrm{sO}_{2}$ é de 95-100\% (DiBARTOLA, 2006). No presente estudo os valores de $\mathrm{sO}_{2}$ variaram de 94,8 a 96,6\% e não houve interação entre tratamento e tempo, nem diferença significativa para a $\mathrm{sO}_{2}$ (Tabela 1$)$.

O ânion gap (AG) é utilizado primariamente para identificar acidose metabólica e confirmar distúrbios mistos e no prognóstico dos pacientes, como citaram GOSSET et al. (1987) e MORAIS \& DiBARTOLA (1993). Houve discreto, mas não-significativo $(\mathrm{P}=0,746)$, aumento nos valores do AG em T1 nos animais de todos os grupos. Esse aumento provavelmente foi ocasionado pela elevação nas concentrações dos ânions nãomensuráveis no plasma, em conseqüência da hipovolemia decorrente do protocolo de desidratação, como citaram FELDMAN \& ROSENBERG (1981), WHITEHAIR et al. (1995) CARLSON (1997) e ANDREWS \& GRINDEM(2000).
Os valores do AG em T2, T3 e T4 nos animais dos grupos RL, RS e GF diminuíram $(\mathrm{P}=0,070)$ (Tabela 1), ficando abaixo dos valores de referência (15-25 $\left.\mathrm{mEqL}^{-1}\right)$, fato decorrente da hidratação, como descreveu CARLSON (1997), MOE \& FUSTER (2003) e DiBARTOLA(2006).

\section{CONCLUSÕES}

A administração intravenosa de soluções eletrolíticas comerciais em cães desidratados experimentalmente ocasiona alterações discretas sobre o equilíbrio ácido-base. A solução Ringer lactato determina efeito alcalinizante, sinalizado por aumento nos valores do $\mathrm{pH}(\mathrm{a}), \mathrm{cHCO}_{3}(\mathrm{aP}), \mathrm{ctCO}_{2}(\mathrm{aP})$ e cBase(a), podendo a mesma ser utilizada no tratamento de animais com acidose metabólica de intensidade discreta a moderada. Por sua vez, as soluções Ringer simples e glicofisiológica originam leve diminuição nos valores de cBAse(a). Por apresentar efeito acidificante, essas soluções podem ser uma opção para tratar cães com alcalose metabólica.

\section{FONTES DE AQUISIÇÃO}

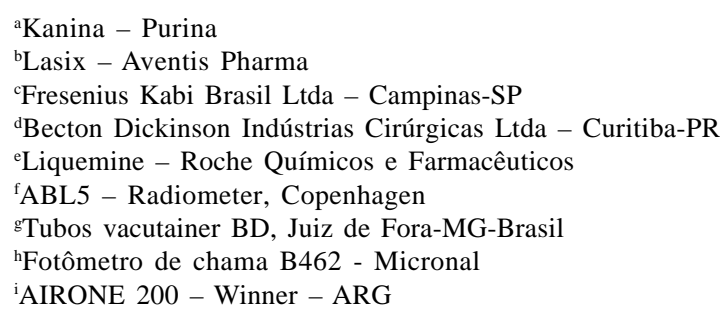

\section{COMITÊ DE ÉTICA E BIOSSEGURANÇA}

O trabalho foi aprovado pela Comissão de Ética e Biossegurança do DVT/UFV - Proc. n 31/2006.

\section{REFERÊNCIAS}

ANDREWS, J.M.; GRINDEM, C.B. Interpreting electrolyte, anion gap, and total carbon dioxide data. Vet Med, v.8, p.548553, 2000.

BREYER, J.; JACOBSON, R.H. Molecular mechanisms of diuretic agents. Annu Rev Med, v.141, p.265-270, 1990.

CARLSON, G.P. Fluid, electrolyte, and acid-base balance. In:_____. Clinical biochemistry of domestic animal. 5.ed. San Diego: Academic, 1997. p.485-516.

CONSTABLE, P. Fluid and electrolyte therapy in ruminants. Vet Clin North Am Food Animal Pract, v.19, p.557-597, 2003.

DiBARTOLA, S.P. Introduction to acid-base disorders. In: Fluid, eletrolyte and acid-base disorders in small animal practice. 3.ed. Missouri: Elsevier, 2006. p.229-251. 
FELDMAN, B.F.; ROSENBERG, D.P. Clinical use of anion and osmolal gaps in veterinary medicine. J Am Vet Med Assoc, v.178, p.396-398, 1981.

FERREIRA, F.M.; PACHALY, J.R. Manual de fluidoterapia em pequenos animais. São Paulo: Guará, 2000. 79p.

FOSTER, S.J. Some aspects of fluid therapy in practice. J Small An Pract, v.11, p.337-357, 1970.

FREESTONE, J.F. et al. Furosemide and sodium bicarbonateinduced alkalosis in the horse and response to oral $\mathrm{KCl}$ or $\mathrm{NaCl}$ therapy. Am J Vet Res, v.50, p.1334-1339, 1989.

GOSSET, K.A. et al. Contribuition of whole blood L-lactate, pyruvate, D-lactate, acetoacetate, and 3-hydroxybutirate concentrations to the plasma anion gap in horses with intestinal disordens. Am J Vet Res, v.48, p.72-75, 1987.

HARTSFIELD, S.M. et al. Sodium bicarbonate and bicarbonate precursor for treatment of metabolic acidosis. J Am Vet Med Assoc, v.179, p.914-916, 1981.

JOHNSON, P.J. Electrolyte and acid-base disturbances in the horse. Vet Clin North Am Equine Pract., v.11, p.491-514, 1995.

KANEKO, J.R. et al. Clinical biochemistry of domestic animal. 5.ed. San Diego: Academic, 1997. 932p.

LEAL, M.L.R. et al. Uso de bicarbonato e lactato-L para correção da acidose metabólica sistêmica em bovinos com acidose láctica ruminal aguda. Arq Bras Med Vet Zoot, v.59, p.971-976, 2007.

LINDEMAN, R.D.; PAPPER, S. Therapy of fluid and electrolyte disorders. Annals Int Med, v.82, p.64-70, 1975.

MATHEWS, K.A. The various types of parenteral fluids and their indications. Vet Clin North Am Small An Pract, v.28, p.483-513, 1998.

MATHEWS, K.A. Monitoring fluid therapy and complications of fluid therapy. In:_____ Fluid, eletrolyte and acidbase disorders in small animal practice. 3.ed. Missouri: Elsevier, 2006. p.337-391.
MOE, O.W.; FUSTER, D. Clinical acid-base pathophysiology: disorders of plasma anion gap. Best Pract Res Clin Endoc Met, v.17, p.559-574, 2003.

MORAIS, H.A.; COSTABLE, P.D. Strong ion approach to acid-base disorders. In: Fluid, eletrolyte and acidbase disorders in small animal practice. 3.ed. Missouri: Elsevier, 2006. p.310-321.

MORAIS, H.S.A.; DiBARTOLA, S.P. Mixed acid-base disorders. Part I. Clinical approach. Comp Cont Educ Pract Vet, v.15, p.1619-1626, 1993.

NAYLOR, J.M.; FORSYTH, G.W. The alkalinizing effects of metabolizable bases in the healthy calf. Can J Vet Res, v.50, p.509-516, 1986.

NELSON, D.L.; COX, M.M. Lehninger princípios de bioquímica. 3.ed. São Paulo: Salvier, 2002. 975p.

RADOSTITIS, O.M et al. Clínica veterinária. Um tratado de doenças dos bovinos, ovinos, suínos, caprinos e eqüinos. 9.ed. Rio de Janeiro: Guanabara Koogan, 2002. 1737p.

RIBEIRO FILHO, J.D. et al. Hemogasometria em eqüinos com compactação experimental do cólon maior tratados com sene, fluidoterapia enteral e parenteral. Ciência Rural, v.37, p.755761, 2007

RUSSELL, K.E. et al. Strong ion difference approach to acidbase imbalances with clinical applications to dogs and cats. Vet Clin North Am Small An Pract, v.26, p.1185-1201, 1996.

SEAHORN, T.L.; CORNICK-SEAHORN, J. Fluid therapy. Vet Clin North Am Equine Pract, v.10, p.517-525, 1994.

WELLMAN, M.L. et al. Applied physiology of body fluids in dogs and cats. In:__. Fluid, eletrolyte and acid-base disorders in small animal practice. 3.ed. Missouri: Elsevier, 2006. p.3-24

WHITEHAIR, K.J et al. Clinical applications of quantitative acid-base chemistry. J Vet Int Med, v. 9, p.1-11, 1995. 